\title{
Emotion Sharing with the Emotional Digital Picture Frame
}

\author{
Kyoung Shin Park ${ }^{1}$, Yongjoo $\mathrm{Cho}^{2}$, Minyoung Kim \\ $\mathrm{Ki}$-Young $\mathrm{Seo}^{4}$, and Dongkeun $\mathrm{Kim}^{2}$ \\ ${ }^{1}$ Department of Multimedia Engineering, Dankook University, Korea \\ ${ }^{2}$ Division of Digital Media, Sangmyung University, Korea \\ ${ }^{3}$ Department of Computer Science, Sangmyung University, Korea \\ ${ }^{4}$ Department of Computer Science, Dankook University, Korea \\ kpark@dankook.ac.kr, ycho@smu.ac.kr, pupleshine@gmail.com, \\ windzard@empas.com, dkimesmu.ac.kr
}

\begin{abstract}
This paper presents the design and implementation of emotional digital picture frame system, which is designed for a group of users to share their emotions via photographs with their own emotional expressions. This system detects user emotions using physiological sensor signals in real-time and changes audio-visual elements of photographs dynamically in response to the user's emotional state. This system allows user emotions to be shared with other users in remote locations. Also, it provides the emotional rule authoring tool to enable users to create their own expression for audio-visual element to fit their emotion. In particular, the rendering elements of a photograph can appear differently when another user's emotion is received.
\end{abstract}

Keywords: Emotional Digital Picture Frame, Emotional Intelligent Contents, Emotional Rule Authoring Tool.

\section{Introduction}

Emotion stimulates five senses of a human being and it plays a critical role while enjoying multimedia contents, such as movie, game and picture. Typically such contents are created by designers beforehand with one or more emotional themes (such as sadness, happiness, suspense and so on). They do not dynamically change its emotional theme in real-time according to user's emotional feeling. However, recent research has emphasized the role of emotion in information technology, and a number of emotion-related studies are underway.

As shown in Fig. 1, Emotionally Intelligent Content (EIC) is the digital content that goes beyond pre-defined emotional theme. For instance, the emotionally intelligent game detects if a user tends to be too immersed in playing a violent game, and it would change its visual and aural aspects to distract the user [1]. Thus, EIC recognizes user emotional states in real-time and responses to them to give certain emotion or make them more engaged into the content. Also, EIC allows each individual user to express or change its visual and aural elements based on user emotional states. 


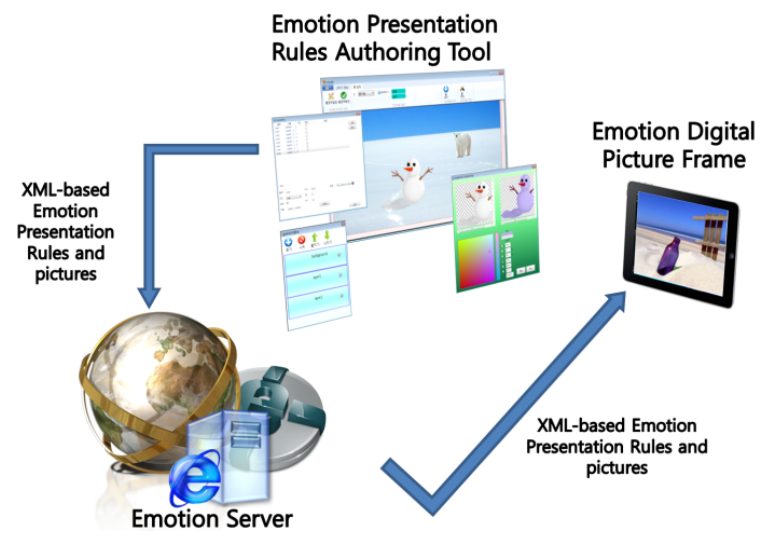

Fig. 1. The Conceptual Diagram of the Emotional Digital Picture Frame

Photographs often capture a user's special memories and moments. Photographs are also great medium for sharing emotion with others. When a family sees a photo of a child riding a bike in a garden, it may trigger a memory for their private thoughts or emotional feeling at the time, even though the details may not be shown in the picture. In this paper we focus on such emotional sharing via an emotional digital picture frame in which the system allows altering the photo's visual and aural presentation customized for each user's preference. In this system, user's own emotion rules specify how the visual and aural properties of the picture such as color, brightness as well as sound would be rendered to other users.

Fig. 1 shows a conceptual diagram of emotional digital picture frame system. As shown in Fig. 1, if a new picture with emotional rules is uploaded to the server, it is automatically shown on other remote users. This system uses PPG, GSR, SKT physiological signals to retrieve user emotional states. The audio-visual elements are then rendered in response to user emotions. Using the Emotion Rule Authoring Module, users can create XML-based emotional rules that define the audio-visual elements to fit their emotions. In this system, changing user emotions can be verified through the picture appearance, such as the saturation, luminosity and brightness. This mechanism allows the remote user to get the other user's emotion in real-time.

We will first look at related work and discuss the design and implementation of emotional digital picture frame system and its authoring tool. Then, we will describe our prototype and analyze the results of the test conducted on the emotion images. Finally we offer our conclusions and discuss steps for future research.

\section{Related Works}

There are many studies on sharing emotion using photo frames. Sondhi and Sloane developed a picture-sharing system for a ubiquitous smart home environment [2]. In this system, users are able to input descriptive emotional words onto the pictures that are shared. 

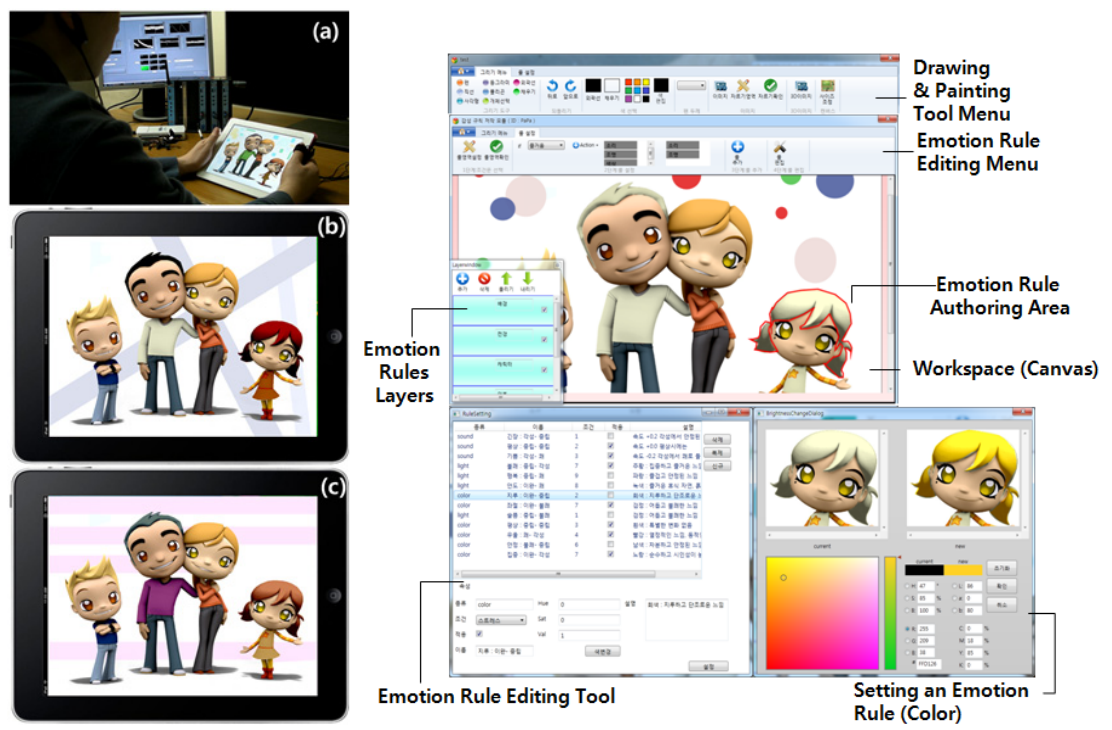

Fig. 2. A Prototype of Emotional Digital Picture Frame with Emotional Rule Authoring Tool

LumiTouch [3] is an emotional communication device which consists of a pair of interactive picture frames. It is used for enhancing the communication between loved ones. In LumiTouch, light-emitting elements and pressure sensors are attached to the photo frame. The sensors then detects when a user took hold of the photo frame with his/her hands, and then different colored lights are displayed on the other user's frame depending on how, how long and where a user holds the frame. Also, when a user is standing in front of the frame, the other LumiTouch device emits ambient light glows to indicate the presence of user. The features of LumiTouch allow users to display their emotions and develop an abstract emotion language for basic communication.

The EmoHeart project by University of Tokyo took Second Life one step further by displaying emotions (via inputted text) on the avatars' chests [4]. Second Life is an online virtual world where user avatars utilize clients to meet and interact. It is used to foster business and personal relationships and as a medium for the exchange of new ideas. The EmoHeart project requires the active analysis of inputted text to discover user emotions, which are then displayed on the avatar's face or as a heart on the avatar's chest.

The Emoti-Picture Frame added a tangible user interface in a frame to convey emotional features to remote users [5]. This frame is divided into two parts: the picture area (on the left) displays the remote user's emotion and the feeling area (on the right) consists of emotional buttons and heart-shaped emotion indicator, which allows the current user to express his/her own emotions. When a user pressed an emotional button, the relevant emotion is transmitted to the other user and the related picture is displayed on the frame along with the heart emotional indicator blinking.

All these systems allow users to share emotions, but they only work for two users and do not provide any infrastructure to make them scalable for more users. Also, the way emotions are understood in these cases have either been pre-determined or a new 
language must be established that both users have agreed to, which makes it difficult for users to adequately express their emotions. Another problem is that emotions must be actively expressed in order to be transmitted. On the other hand, our proposed emotional digital picture frame system not only allows for the sharing of user emotions, but utilizes physiological signals so that emotions can be transmitted and shared without any work on the part of the user. It also allows users to express and understand emotions in their own individualized way.

\section{Emotional Digital Picture Frame}

Each person has a unique personality and often shows different reactions to the same contents. As such, a uniform emotion response is not likely to satisfy all users [6]. Schneiderman[7] also pointed out that users preferred interactions that they were able to control themselves. In this research we introduce an emotional picture frame system that responds to individual user emotions by user-specified emotional rules. Unlike previous works, this system enables for users to be able to directly control how audio-visual elements on a picture will change depending on their emotions.

Fig. 2 shows a prototype of the emotional digital picture frame system built for sharing photographs among group members with individualized emotion. We conjured up a scenario of a family separated by distance. In particular, we envisioned this system allowing a father separated from his family to be able to share emotions. Once the father touches the frame, his emotional state is read by the sensors on the frame and his emotion is then displayed on the family's picture frame. Conversely, a daughter touches her picture frame to project her emotional states onto the father's frame.

In this current prototype, a user attaches the sensors to his/her finger and ear to view the emotional picture as shown in Fig. 2 (a). While he/she is looking at the picture, the emotions he/she feels are transmitted to the remote user via the server. The remote user is looking at the same picture that is slightly altered according to his/her emotion rules. Fig. 2 (b) and (c) show how the individualized emotion rules affect the screen differently. Fig. 2 (b) shows the picture of the daughter on the father's screen. Fig. 2 (c) shows the father's emotional state. As we can see, the color of the daughter's hair and clothes as well as the background image appears different from each other's view. Fig. 2 (d) shows the emotional rule authoring tool to customize individual's emotional expression.

\section{Design and Implementation}

Fig. 3 shows the system architecture of the emotional digital picture frame system. This system is built on top of Emotional Content Framework that consists of the Emotion Recognizer Module, the Emotion Server, the Emotional Rule Authoring Module and the Emotional Contents Player Module. 


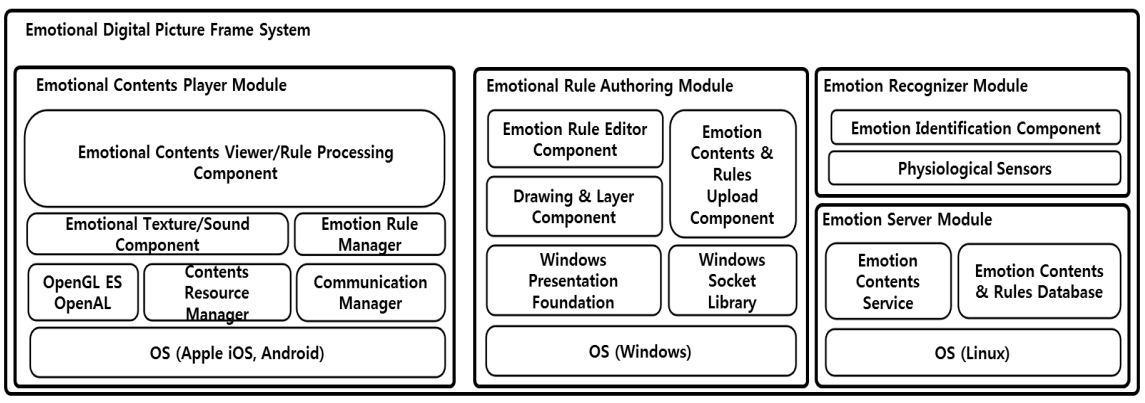

Fig. 3. The System Architecture of the Emotional Digital Picture Frame

\subsection{Emotion Recognizer Module}

The Emotion Recognizer Module receives user's physiological signals via PPG (pulse wave), SKT (skin temperature), and GSR (skin resistance) sensors attached to the BIOPAC MP100 system. It then applies the emotion deduction algorithm on the signals using our own LabView (Windows platform) program [CE] to classify into one of the nine emotional states: alertness, unpleasant, pleasant, relaxed, stressed, excited, sluggish, tired or neutral, in a two-dimensional space based on Russell's emotion model [8].

\subsection{Emotion Server}

The Emotion Server is a REST (Representational State Transfer)-based service running on a Linux-based Apache web server. It manages user's emotional states (retrieved from the Emotion Recognizer Module) and the emotional rules suited for each individual and the contents he/she created (using the Emotional Rule Authoring Module) stored on the MySQL database system. In order to do this, a unique identification number is given to each user along with the user's emotional states at given dates and times. The server also notifies the current user's emotional states to Emotional Digital Picture Frame clients.

\subsection{Emotional Rule Authoring Tool}

The Emotional Rule Authoring Tool is used for creating individualized emotional rules. This module is implemented using $\mathrm{C \#}$ and Windows Presentation Foundation (WPF) library. Users login with their identification number to update their individualized emotion rules in personalized spaces in the server. When users upload new pictures, sharing (among Emotional Digital Picture Frame systems) commences. These shared pictures reflect the emotion rules that were saved for the user. If no emotion rules were specified, the server applies default emotion settings. Fig. 2 (d) shows specifying the emotional rules by selecting the field in the workspace where the rules will be applied. The emotional rule field is where a user can alter color, saturation and light luminosity and overall brightness on the image to reflect individualized emotion. Once the field is selected, a user selects the emotion response 
from the toolbox to specify the emotion rules. This authoring tool provides several primary drawing features for easy manipulation of rules on the picture and layers for defining more complex rules.

\subsection{Interaction Scenario}

The Emotional Contents Player Module displays emotional pictures on devices like the Apple iOS and Google Android. It receives new pictures and emotion rules on the server and then renders the customized visual and aural aspects on an image in response to user's emotional states. This module renders graphics using OpenGL ES 3D mobile-platform graphics library and sounds using the OpenAL library. The libcURL library was used for communication between the Emotional Contents Player Module and the Emotion Server to make use of the various Web service features. In addition, open source libXML was used to handle XML for emotion rules and contents expression.

\section{Conclusions}

This paper introduces a prototype of the Emotional Digital Picture Frame system constructed with Emotional Content Framework designed for creating emotional pictures by using individualized emotion rules. In this prototype, user's emotions are read by analyzing physiological sensors and these emotions are sent to remote users via the server. The picture changes its color and lighting dynamically depending on the specified emotion rules according to user emotions. In this prototype, users can utilize the Emotional Rule Authoring Tool to specify individualized emotion rules. In future research, we plan to conduct a systematic experiment to evaluate how much the users would be affected by sharing emotional pictures. Also, we will improve other elements to determine the effect of emotion contents and emotion sharing features.

Acknowledgements. This research was supported by Basic Science Research Program through the National Research Foundation of Korea (NRF) funded by the Ministry of Education, Science and Technology (Grant Number: 2012R1A1A1010815).

\section{References}

1. Kim, M., Park, K.S., Cho, Y.: Design and Implementation of the iOS-based Game Framework for Emotional Character Expression. Journal of Korean Society for Computer Game 24(1) (March 2011)

2. Sondhi, G., Sloane, A.: Digital Photo Sharing and Emotions in a Ubiquitous Smart Home. In: Venkatesh, A., Gonsalves, T., Monk, A., Buckner, K. (eds.) Home Informatics and Telematics: ICT for the Next Billion. IFIP, vol. 241, pp. 185-200. Springer, Boston (2007)

3. Chang, A., Resner, B., Koerner, B., Wang, X., Ishii, H.: LumiTouch: an emotional communication device. In: Extended Abstracts on ACM Human Factors in Computing Systems, CHI (2001) 
4. Neviarouskaya, A., Prendinger, H., Ishizuka, M.: EmoHeart: Conveying Emotions in Second Life Based on Affect Sensing from Text. In: Advanced in Human-Computer Interaction (2010)

5. Neyem, A., Aracena, C., Collazos, C.A., Alarcón, R.: Designing Emotional Awareness Devices: What One Sees is What One Feels. Revista Chilena de Ingenieria 15(3), 227-235 (2007)

6. Richard, S.: Lazarus, Emotion and Adaptation, pp. 15-29. Oxford University Press (1991)

7. Schneiderman, B.: Designing the User Interfaces: Strategies for Effective Human-Computer Interaction, 3rd edn. Addison-Wesley, Reading (1998)

8. Posner, J., Russell, J.A., Peterson, B.S.: The circumplex model of affect: An integrative approach to affective neuro science, cognitive development and psychopathology, vol. 17, pp. 715-734. Cam bridge University Press (2005) 